\section{Prevalência de defeitos de esmalte e sua relação com cárie dentária nas dentições decídua e permanente, Indaiatuba, São Paulo, Brasil}

\author{
Prevalence of enamel defects and the relationship \\ to dental caries in deciduous and permanent \\ dentition in Indaiatuba, São Paulo, Brazil
}

\author{
${ }^{1}$ Faculdade de Odontologia \\ de Piracicaba, Universidade \\ Estadual de Campinas, \\ Piracicaba, Brasil. \\ 2 Centro de Ciências da Vida, \\ Pontifícia Universidade \\ Católica de Campinas, \\ Campinas, Brasil. \\ Correspondência \\ M. L. R. Sousa \\ Departamento de \\ Odontologia Social \\ Faculdade de Odontologia \\ de Piracicaba, Universidade \\ Estadual de Campinas. \\ Av. Limeira 901, Piracicaba, SP \\ 13414-900, Brasil. \\ luzsousa@fop.unicamp.br
}

\begin{abstract}
The aim of this study was to determine the prevalence of hypoplasia, demarcated opacity and dental fluorosis among schoolchildren with deciduous and permanent dentition. The association between enamel defects and dental caries was also verified. The sample consisted of 624 schoolchildren aged 5 and 309 aged 12. The dmft and DMFT indexes were used to assess dental caries prevalence, DDE to assess enamel defects, and Dean to assess fluorosis. Chi-squared test was used to test significance $(p<0.05)$ and odds ratio to analyze prevalence of dental caries and enamel defects. A positive association between dental caries and enamel defects (hypoplasia, demarcated opacity and dental fluorosis) was observed for schoolchildren aged 5. However, only hypoplasia and demarcated opacity were associated with caries experience in permanent dentition. The results of this study indicated that children had increased odds of dental caries when enamel defect was present, both in deciduous and permanent dentition; further studies are needed to give evidence to this association.
\end{abstract}

Dental Enamel Hypoplasia; Dental Fluorosis; Dental Caries; Oral Health
Rosana H. Schlittler Hoffmann 1

Maria da Luz R. de Sousa 1

Silvia Cypriano 2

\section{Introdução}

Além dos fatores já conhecidos e muito estudados, tem sido sugerido, na literatura, que os defeitos de esmalte também poderiam contribuir para uma maior incidência da cárie dentária 1,2 .

O esmalte dentário é um tecido incomum que, uma vez formado, não sofre remodelação como outros tecidos duros. Por causa de sua natureza não remodeladora, alterações durante sua formação são permanentemente registradas na superfície dentária.

Sabe-se que a formação do esmalte dentário pode ser dividida em três estágios: (i) o estágio da formação da matriz, no qual as proteínas envolvidas na amelogênese são produzidas; (ii) o estágio da calcificação, no qual é depositado mineral, e a maior parte das proteínas originais são removidas; e (iii) o estágio da maturação no qual o esmalte recém mineralizado sofre processo final de calcificação, e as proteínas ainda remanescentes são removidas. Esses processos acontecem sob influência genética e mudanças ambientais, dessa forma, o desenvolvimento de defeitos de esmalte pode resultar de qualquer dano ocorrido nesses estágios.

Geralmente é aceito que distúrbios que ocorram durante os primeiros estágios de desenvolvimento do esmalte resultarão na redução da quantidade ou espessura do esmalte, ou seja, a hipoplasia do esmalte ${ }^{3}$. Hipoplasia de esmalte pode ser, então, definida como deficiência na 
quantidade do esmalte, resultante de alterações do desenvolvimento, e pode ocorrer na forma de fóssulas, fissuras ou perdas de áreas grandes de esmalte.

Em contraste, distúrbios ocorridos durante o estágio da calcificação e maturação do desenvolvimento do esmalte podem levar a deficiências na mineralização (hipocalcificação) e geralmente se manifestam como mudanças na translucência ou opacidades do esmalte. As opacidades podem ser demarcadas ou difusas. A opacidade demarcada apresenta um esmalte de espessura normal e com uma superfície intacta, porém existe uma alteração na translucidez do esmalte, de grau variável. Essa translucidez é demarcada a partir do esmalte adjacente normal com limites nítidos e claros, podendo apresentar uma coloração branca, bege, amarela ou marrom. A opacidade difusa também é uma anormalidade envolvendo uma alteração na translucidez do esmalte, de grau variável, e de coloração variável como a opacidade demarcada. Todavia, não existe um limite claro entre o esmalte normal adjacente e a opacidade difusa, podendo apresentar-se clinicamente de forma linear ou em placas, ou ter uma distribuição confluente ${ }^{4}$. Esse tipo de defeito tem sido identificado como fluorose dentária por alguns autores, sendo adotada essa terminologia no presente trabalho.

Como o esmalte dentário pode ser afetado por muitos fatores, as mudanças provocadas nele podem fornecer indícios como, por exemplo, sobre o tempo e a natureza desses eventos. Desse modo, defeitos de esmalte podem, então, ser estudados como um marcador de muitos eventos biológicos adversos ocorridos durante sua fase de desenvolvimento, e isso pode ter aplicações em investigações clínicas, epidemiológicas e antropológicas.

Assim, considerando que vários estudos epidemiológicos têm apontado uma relação entre a presença de hipoplasia e opacidade demarcada como um risco aumentado de desenvolver cárie dentária $1,2,5,6$, torna-se importante que esse conhecimento seja aprofundado, sendo um marcador importante também para a definição de ações em saúde pública, dependendo da prevalência e severidade que esses defeitos apresentam em diferentes grupos populacionais.

Em acréscimo, tem sido relatado que a presença de hipoplasia de esmalte pode ser considerada um fator de risco de cárie tão relevante quanto a infecção precoce por Streptococcus mutans e a presença de dieta cariogênica 7, pois, se considerarmos que esse tipo de defeito de esmalte compromete sua estrutura em quantidade, ou seja, forma nichos de retenção de placa dental, pode ocorrer uma maior probabilidade da instalação e da progressão da doença cárie dentária.

Ellwood \& O'Mullane 8, estudando uma população no norte do País de Gales com baixos níveis de flúor na água de abastecimento público, observaram relações inversas entre a presença de cárie dentária e opacidade difusa e demarcada. Foram avaliadas crianças com idade média de 12 anos, e o grupo de crianças com a presença de opacidade difusa teve uma média CPOS de 2,0, apresentando uma prevalência de cárie $33 \%$ menor quando comparado ao grupo sem essa alteração (CPOS = 3,0). Em contraste, as crianças que apresentavam opacidades demarcadas tiveram um CPOS de 3,2, representando uma prevalência de cárie dental $28 \%$ menor, quando comparadas com as crianças que não apresentavam essa alteração (CPOS $=2,5)$. O presente estudo foi realizado nas dentições decídua e permanente, sendo que a literatura traz poucos dados em relação à dentição decídua.

Desse modo, considerando que poucos estudos agregam informações epidemiológicas sobre a prevalência de defeitos de esmalte na dentição decídua, assim como a carência de estudos de base nacional e a relevância atual sobre esse tema, os objetivos deste estudo foram: (i) verificar a prevalência de hipoplasia, opacidade demarcada e fluorose dentária no Município de Indaiatuba, São Paulo, Brasil, no ano de 2004, nas dentições decídua e permanente; e (ii) verificar a associação da presença desses defeitos de esmalte e a cárie dentária.

\section{Material e método}

O presente estudo é parte integrante de uma pesquisa mais ampla sobre cárie dentária, realizada em 38 municípios da região de Campinas e Piracicaba, Estado de São Paulo, Brasil, e foi executado após a aprovação do Comitê de Ética em Pesquisa da Faculdade de Odontologia de Piracicaba (n. 155/2004) e obtenção das autorizações dos responsáveis para que as crianças participassem do estudo.

A cidade de Indaiatuba é considerada de médio porte (172.140 habitantes), apresentando, em 2005, um índice de desenvolvimento humano (IDH) de 0,83. A taxa de urbanização da cidade é de $98,7 \%$, e a de atendimento de abastecimento de água, de 94,37\%, segundo dados obtidos da Fundação Sistema Estadual de Análise de Dados - SEADE (http://www.seade.gov.br, acessado em 20/Out/2005). A água de abastecimento público do município vem sendo fluoretada há 26 anos.

A amostra deste estudo foi probabilística. Para o cálculo do tamanho da amostra para a den- 
tição decídua, foram utilizados o valor da média ceod (ceod $=1,79$ ) e desvio padrão ( $\mathrm{DP}=3,09$ ), obtidos em um estudo anterior realizado no ano de 2000, adotando-se, para tal, nível de confiança de $95 \%$, precisão de $20 \%$ e efeito do desenho (deff) de 2 . Somaram-se $20 \%$ a esse total, a fim de compensar eventuais perdas e recusas, resultando em um tamanho amostral de 624.

Para o cálculo do tamanho da amostra para a dentição permanente, foram utilizados o valor da média (CPOD foi de 1,84 aos 12 anos) e DP =2,13, obtidos em um estudo realizado no ano de 2000 , adotando-se, para tal, nível de confiança de 95\%, precisão de $20 \%$ e efeito do desenho (deff) de 2 . Somaram-se $20 \%$ a esse total, a fim de compensar eventuais perdas e recusas, resultando em um tamanho amostral de 309.

O sorteio das instituições de ensino teve como base as escolas particulares, municipais e estaduais fornecidas pela Secretaria de Educação, freqüentadas por crianças de cinco anos e, para as de 12 anos, apenas as de escolas públicas. Procedeu-se ao sorteio ponderado, de tal forma que as escolas com maior número de alunos tiveram mais chances de serem sorteadas, seguindo as recomendações adotadas no Levantamento Nacional SB Brasil 2000 9. O sorteio dos elementos amostrais também seguiu essas recomendações.

A calibração da equipe, formada por dois cirurgiões dentistas, foi realizada durante 36 horas, divididas entre discussão teórica das variáveis utilizadas, códigos e critérios de exame e discussão prática, simulando as diferentes condições e situações que os profissionais encontrariam durante a realização do trabalho prático. A porcentagem de concordância interexaminadores para a cárie foi de $96 \%$, e quanto à concordância intra-examinadores tanto para cárie quanto para fluorose dentária, ambas se apresentaram acima do nível aceitável (85\%).

O exame epidemiológico foi realizado sob luz natural, com o examinador e o paciente sentados, usando espelho bucal plano número $5 \mathrm{e}$ sonda periodontal (ball point). Os dentes foram avaliados sem serem secos.

Para avaliação da cárie dentária, utilizaramse os índices ceod para dentição decídua e CPOD para dentição permanente, segundo os critérios de diagnóstico da Organização Mundial da Saúde (OMS) 4 .

Os critérios de diagnóstico para os defeitos de esmalte utilizados foram: índice de defeitos de desenvolvimento do esmalte modificado (DDE), para identificar as opacidades demarcadas e hipoplasia, e o índice de Dean para a fluorose dentária, sendo ambos os índices recomendados pela OMS 4. Durante a avaliação, foi anotado se a criança apresentava algum tipo de defeito de esmalte em pelo menos uma superfície dental.

O índice DDE classifica os defeitos de esmalte em três principais categorias: opacidades demarcadas, opacidades difusas e hipoplasias, porém, no presente estudo, as opacidades difusas não foram consideradas por esse índice, e, assim, a presença de fluorose dentária foi considerada pelo índice de Dean 4 a partir do grau 1.

As opacidades demarcadas estão presentes quando existe uma anormalidade na translucência do esmalte, mas a espessura do esmalte não é afetada. Esse tipo de opacidade apresenta margens bem definidas entre o esmalte afetado e o esmalte normal, podendo apresentar variações na coloração da superfície dentária, variando da cor branca à cor creme, amarela ou até marrom. Alguns defeitos são translucentes na superfície enquanto outros podem apresentar falta de brilho na sua aparência ${ }^{10}$. Fluorose dentária parece branca quando o dente erupciona e apresenta uma variação similar aos defeitos demarcados na translucência, porém a principal diferença entre as opacidades difusas e as demarcadas é que as difusas não apresentam uma margem que claramente as definem do esmalte adjacente normal. As lesões de fluorose geralmente se encontram dentro desse tipo de defeito ${ }^{10}$. No presente estudo, consideraram-se os critérios do índice de Dean, em que a fluorose apresenta-se de forma bilateral e simétrica, com padrão estriado horizontal cruzando os dentes, podendo ainda ser em placas ou ter distribuição confluente.

Defeitos hipoplásicos estão associados com a perda da integridade da superfície do esmalte 10.

A estatística descritiva foi realizada para verificar a prevalência de defeitos de esmalte (hipoplasia, opacidade demarcada e fluorose dentária) aos cinco anos (dentição decídua) e 12 anos (dentição permanente) de idade. O critério utilizado para definir o desfecho experiência de cárie na dentição decídua foi ceod $>0$, enquanto o critério utilizado para definir o mesmo desfecho na dentição permanente foi CPOD $>0$. Por tratar-se de um estudo transversal exploratório, a associação significativa entre defeitos de esmalte e experiência de cárie foi verificada por meio de análise bivariada (teste qui-quadrado), considerando o valor para rejeição da hipótese nula de $\mathrm{p}<0,05$. Para análise da força e direção dessa associação, utilizou-se a razão de chances ou a odds ratio (OR). O programa utilizado para a realização das análises estatísticas foi o Epi Info (Centers for Disease Control and Prevention, Atlanta, Estados Unidos). 


\section{Resultados}

Durante os exames clínicos epidemiológicos, a porcentagem de concordância intra-examinadores, tanto para a cárie dentária quanto para os defeitos de esmalte, esteve acima do nível aceitável de $85 \%$.

As crianças participantes deste estudo apresentavam um nível sócio-econômico relativamente homogêneo, pois, entre as crianças de cinco anos, 96,2\% freqüentavam escolas públicas $(n=600)$, e 3,8\%, escolas particulares $(n=24)$, enquanto que, aos 12 anos, $100 \%$ delas provinham de escolas públicas.

A amostra, segundo o gênero, apresentou-se dividida da seguinte forma: aos cinco anos, $51 \%$ eram do gênero masculino ( $\mathrm{n}=318$ ), e $49 \%$, do gênero feminino ( $\mathrm{n}=306)$; aos 12 anos, 45,6\% eram do gênero masculino ( $\mathrm{n}=141)$, e $54,4 \%$, do feminino ( $n=168)$, não se verificaram diferenças estatisticamente significantes entre a prevalência de defeitos de esmalte em relação ao gênero em nenhuma das idades ( $p>0,05)$.

As prevalências para os três tipos de defeitos de esmalte (hipoplasia, opacidade demarcada e fluorose dentária) em relação às dentições decídua e permanente podem ser observadas na Tabela 1. Considerando a dentição decídua e permanente, não foram observadas diferenças estatisticamente significantes entre a prevalência de opacidade demarcada e hipoplasia. O defeito de esmalte mais prevalente na dentição decídua foi a opacidade demarcada (20,9\%), enquanto que na dentição permanente foi a fluorose dentária $(26,2 \%)$.

Na dentição decídua, foi maior $(p<0,05)$ a prevalência de hipoplasia, opacidade demarcada e fluorose no grupo de crianças com experiência de cárie (ceod $>0$ ), em relação ao grupo sem experiência de cárie $(\operatorname{ceod}=0)$, conforme a Figura 1.

Para a dentição permanente, ao serem comparados os grupos com experiência de cárie $(\mathrm{CPOD}>0)$ e sem experiência de cárie (CPOD $=0$ ), a prevalência foi maior apenas para os defeitos hipoplásicos e opacidades demarcadas, não diferindo em relação à fluorose dentária (Figura 2).

A Tabela 2 mostra a associação entre cárie e defeitos de esmalte por meio da interpretação dos resultados pela razão de chances ou a OR, além de comparar a prevalência de cárie e as médias dos índices ceod e CPOD no grupo de crianças que apresentavam ou não defeitos de esmalte. Pode-se observar que, na presença de hipoplasia, a chance de as crianças terem cárie dentária na dentição decídua foi 4,8 vezes maior e 11,0 vezes maior na dentição permanente. Em relação às opacidades demarcadas, a chance foi de 1,96 para as crianças com dentição decídua e 3,5 vezes maior para a dentição permanente. Em relação à fluorose dentária, a chance de ter cárie dentária foi de 2,0 vezes maior para a dentição decídua, o que não foi observado para a dentição permanente.

\section{Discussão}

Os resultados deste estudo devem ser interpretados cuidadosamente, pois não foi aferido o percentual de concordância intra e interexaminadores em relação às opacidades demarcadas e hipoplasia; entretanto aferiu-se a concordância intra-examinadores da fluorose dentária, atingindo valores aceitáveis. Sabendo-se que a fluorose geralmente se apresenta de forma mais heterogênea em função da diversidade de graus

Tabela 1

Ocorrência de defeitos de esmalte nas dentições decídua (cinco anos) e permanente (12 anos) de escolares de Indaiatuba, São Paulo, Brasil, 2004

\begin{tabular}{|c|c|c|c|c|c|}
\hline \multirow[t]{2}{*}{ Tipo de defeito } & \multirow{2}{*}{$\begin{array}{c}\text { Ocorrência de } \\
\text { defeitos de esmalte }\end{array}$} & \multicolumn{2}{|c|}{ Dentição permanente } & \multicolumn{2}{|c|}{ Dentição decídua } \\
\hline & & $n$ & $\%$ & $\mathrm{n}$ & $\%$ \\
\hline \multirow[t]{2}{*}{ Opacidade demarcada } & Não & 494 & 79,2 & 247 & 79,9 \\
\hline & Sim & 130 & 20,9 a & 62 & 20,1 a \\
\hline \multirow[t]{2}{*}{ Hipoplasia } & Não & 570 & 91,3 & 292 & 94,5 \\
\hline & Sim & 54 & 8,7 a & 17 & 5,5 a \\
\hline \multirow[t]{2}{*}{ Fluorose dentária } & Não & 517 & 82,9 & 228 & 73,8 \\
\hline & Sim & 107 & 17,1 a & 79 & $26,2 b$ \\
\hline
\end{tabular}

Valores nas linhas seguidos de letras diferentes diferem entre si estatisticamente $(p<0,05)$ (teste qui-quadrado). 
Figura 1

Porcentagem de crianças com defeitos de esmalte segundo o grupo de crianças sem experiência de cárie (ceod = 0) e grupo com experiência de cárie (ceod > 0) na dentição decídua. Indaiatuba, São Paulo, Brasil, 2004

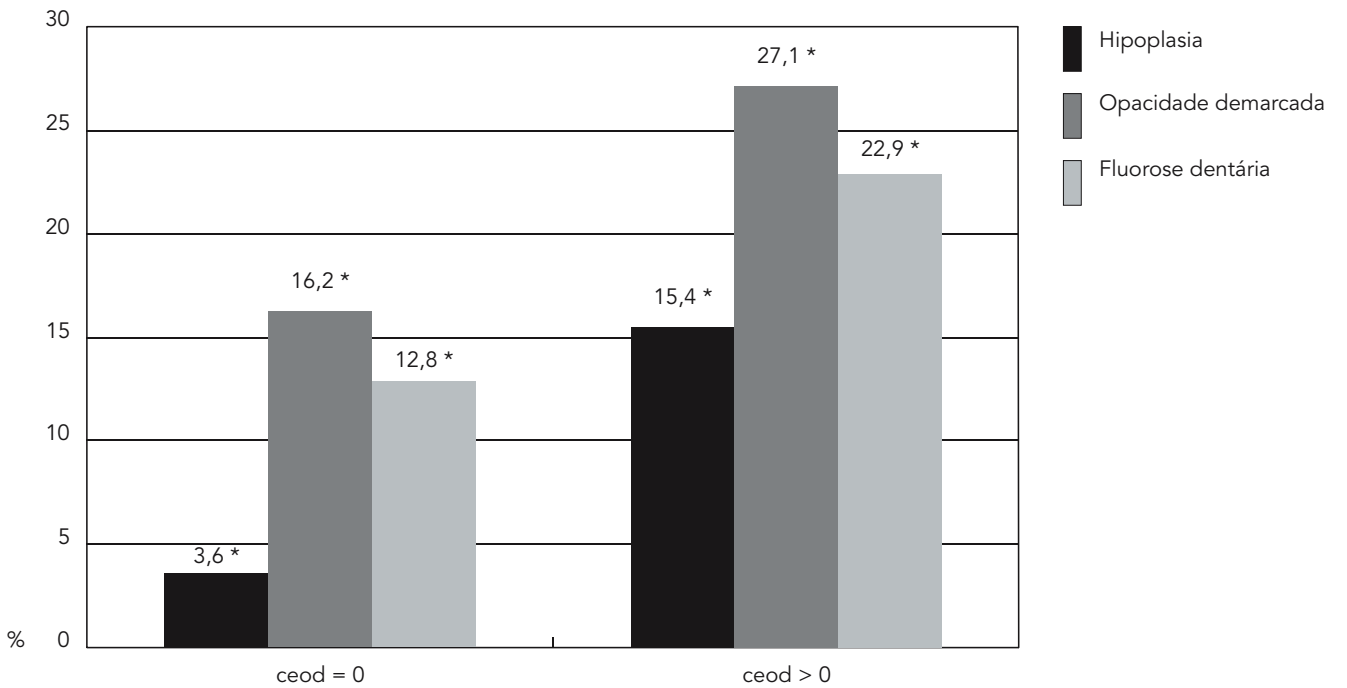

* Valores estatisticamente significantes, segundo teste qui-quadrado, sendo os seguintes valores de $p$ : hipoplasia $(p=0,000)$, opacidade demarcada $(p=0,000)$, fluorose dentária $(p=0,001)$.

Figura 2

Porcentagem de crianças com defeitos de esmalte segundo o grupo de crianças sem experiência de cárie (CPOD = 0) e grupo com experiência de cárie (CPOD > 0) na dentição permanente. Indaiatuba, São Paulo, Brasil, 2004.

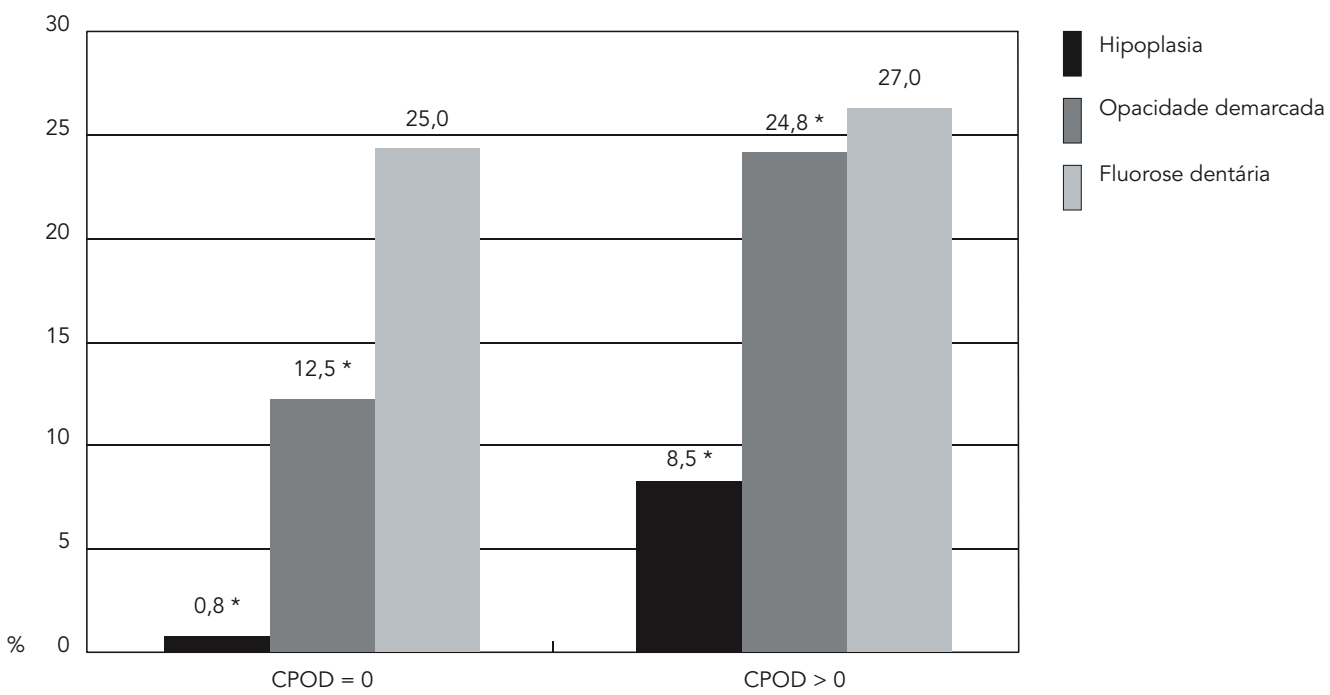

\footnotetext{
* Valores estatisticamente significantes, segundo teste qui-quadrado, sendo os seguintes valores de p: hipoplasia ( $p=0,009)$, opacidade demarcada $(p=0,012)$, fluorose dentária $(p=0,799)$.
} 
Índices ceod e CPOD em relação à presença e ausência de defeitos de esmalte com suas respectivas odds ratio (OR) na presença de defeitos para as dentições decídua e permanente. Indaiatuba, São Paulo, Brasil, 2004

\begin{tabular}{|c|c|c|c|c|c|c|c|c|}
\hline \multirow[t]{2}{*}{ Tipo de defeito } & \multicolumn{3}{|c|}{ Dentição permanente } & \multirow[b]{2}{*}{$\mathrm{p}$} & \multicolumn{4}{|c|}{ Dentição decídua } \\
\hline & Cárie (\%) & ceod & OR & & Cárie (\%) & CPOD & OR & $p$ \\
\hline Hipoplasia $=0$ & 39,5 & 1,41 & 1,00 & & 59,2 & 2,28 & 1,00 & \\
\hline Hipoplasia = 1 & 75,9 & 3,83 & $4,84(2,44-9,73)$ & $<0,001$ & 94,1 & 6,24 & $11,01(1,51-225,57)$ & 0,004 \\
\hline \multicolumn{9}{|l|}{ Opacidade } \\
\hline demarcada $=0$ & 39,3 & 1,45 & 1,00 & & 57,5 & 2,25 & 1,00 & \\
\hline \multicolumn{9}{|l|}{ Opacidade } \\
\hline demarcada $=1$ & 55,8 & 2,32 & $1,96(1,30-2,95)$ & $<0,001$ & 75,4 & 3,47 & $2,32(1,18-4,59)$ & 0,008 \\
\hline \multicolumn{9}{|l|}{ Fluorose } \\
\hline dentária = 0 & 39,7 & 1,44 & 1,00 & & 60,5 & 2,44 & 1,00 & \\
\hline \multicolumn{9}{|l|}{ Fluorose } \\
\hline dentária = 1 & 53,0 & 2,52 & $2,02(1,30-3,40)$ & $<0,001$ & 63,0 & 2,65 & $0,99(0,57-1,74)$ & 0,799 \\
\hline
\end{tabular}

Tipo de defeito: 0 significa ausência do defeito e 1 presença do defeito.

e aparência clínica, os autores acreditam que os resultados do presente estudo devem estar consistentes.

As comparações dos resultados do presente estudo, cuja característica é um estudo transversal de base populacional, com outros estudos, disponíveis na literatura e discutidos a seguir, devem ser feitas com cautela devido às diferenças no delineamento amostral, influências ambientais e diferenças metodológicas, pois a maioria dos estudos foram desenvolvidos a partir de amostras específicas, como crianças prematuras, de baixo peso, com história médica de desnutrição e crianças celíacas, por exemplo.

Assim, quanto à prevalência de hipoplasia encontrada na dentição decídua (8,7\%), esta foi similar a outros estudos encontrados na literatura: $6 \%$ em Iowa, Estados Unidos 11, porém menor que outros; $22,3 \%$ na China ${ }^{6}$ e $22,7 \%$ na Tailândia 5. No estudo realizado com 698 crianças de 4-5 anos em Iowa 11, local com água fluoretada, os autores concluíram que a prevalência encontrada para hipoplasia (6\%) foi baixa comparada com a maioria dos outros estudos, e uma possibilidade para tal resultado é o fato de subestimação do defeito em razão, principalmente, da exclusão dos dentes com coroas de aço inoxidável que teriam sido colocadas como tratamento para cárie ou mesmo para hipoplasia de esmalte.

Em um estudo transversal realizado na China 6 ( $\mathrm{n}=1.344$; 3-5 anos) onde testou-se a associação entre hipoplasia de esmalte e cárie dentária na dentição decídua, encontrou-se que crianças com hipoplasia de esmalte apresentaram maior prevalência de cárie $(92,8 \%)$ do que crianças que não apresentaram hipoplasia (79\%) ( $p<0,001)$, resultados esses semelhantes aos encontrados no presente estudo, diferindo apenas em relação aos valores percentuais (Tabela 2), que foram menores provavelmente em função de diferenças nas médias de idades entre os dois estudos. A análise estatística multivariada do estudo chinês indicou que a presença de hipoplasia, idade, tempo de residência e amamentação foram também variáveis significantes que afetavam a variação do desfecho cárie.

Em um outro estudo, realizado por Kanchanakamol et al. 5 que avaliaram a prevalência dos defeitos de desenvolvimento do esmalte e cárie dental em 344 crianças com idades entre 1 a 4 anos de uma comunidade agrícola de baixo nível sócio-econômico da Tailândia, setenta dessas crianças apresentaram desnutrição crônica, e 9,3\%, aguda. Foram examinados apenas os quatro incisivos superiores. Os resultados mostraram que $32 \%$ das crianças examinadas apresentavam defeitos de desenvolvimento de esmalte (hipoplasia e ou opacidades) em um ou mais incisivos. A prevalência da cárie dental foi de $31,9 \%$. O efeito da nutrição no desenvolvimento dos dentes, como também no desenvolvimento de cáries, é geralmente aceito na literatura 12. Muitos nutrientes específicos têm sido associados com a formação, a calcificação 
e erupção, sendo que, clinicamente, indivíduos que experimentaram uma deficiência severa nutricional têm mostrado também dentes defeituosos em relação à coloração, estrutura, textura e na espessura da superfície do esmalte 13,14 . Tais defeitos colocam esses dentes a um risco maior para o desenvolvimento de cárie após os mesmos terem erupcionado no ambiente oral 6. O estudo realizado na cidade de Indaiatuba não avaliou a situação nutricional das crianças examinadas. Todos os dentes foram avaliados tanto para cárie como para defeitos de esmalte, encontrando-se uma prevalência maior de cárie $(42,6 \%)$ nos indivíduos que apresentavam defeitos. Apesar de o presente estudo apresentar um desenho diferente do de Kanchanakamol et al. 5, a associação entre cárie dental e hipoplasia de esmalte foi significativamente maior que as opacidades e o esmalte sadio em ambos os estudos, sugerindo que a presença de hipoplasia de esmalte resulta num acréscimo da suscetibilidade à cárie dental.

Quanto à prevalência de opacidades demarcadas encontradas na dentição decídua (20,9\%), esta foi maior que a encontrada na China 15 $(1,6 \%)$ e na Tailândia 5 (9,3\%), mas inferior ao valor percentual encontrado em Iowa 11 (27\%). Entretanto, quando se avaliou a prevalência quanto ao gênero nos dois estudos, o de Iowa 11 apresentou diferença significante quanto ao gênero (sexo masculino: $31 \%$, sexo feminino: $23 \%$; $\mathrm{p}=0,018$ ), o mesmo não ocorrendo para o presente estudo $(p=0,12)$. Esse percentual menor para o sexo feminino talvez possa ser explicado, em parte, pela indicação de coroas de aço que os autores relatam, subestimando a prevalência de defeitos.

Em relação à prevalência de fluorose dentária na dentição decídua (11\%), esta foi maior que a encontrada na cidade de Piracicaba $(2,6 \%) 16$ em crianças de cinco anos, sendo que, neste último estudo, foi realizado um censo. Ambos os estudos apresentaram a mesma metodologia para coleta e análise de dados. Simultaneamente à comprovada tendência de declínio da cárie como efeito do uso intensivo de flúor, uma tendência inversa de aumento das taxas de fluorose dentária tem sido observada nos últimos anos. Relatos recentes sobre esse aumento em várias regiões do mundo, tanto em comunidades que consomem água fluoretada como naquelas que consomem água não fluoretada, confirmam essa tendência 16,17,18. Entretanto, Mackay \& Thomson 19 não comprovaram tal aumento e reforçaram os benefícios que a fluoretação das águas de abastecimento pode apresentar como medida de saúde pública, pois crianças expostas continuamente à fluoretação das águas de abastecimento apresentaram $50 \%$ menos experiência de cárie quando comparadas com aquelas não expostas. O principal fator de risco associado a maior prevalência de fluorose mencionado na literatura é o aumento da ingestão média de fluoretos, de variadas formas. O uso de água fluoretada, o uso de dentifrício fluoretado, o uso de suplementos com o flúor e o uso de bebidas ou alimentação infantil em pó contendo fluoretos, antes dos seis anos de idade, têm sido considerados os mais importantes fatores de risco 20 . Por outro lado, é descrito também na literatura que outros fatores que não o fluoreto podem estar induzindo fluorose dentária como o alto consumo de amoxicilina em crianças apresentando infecções, principalmente a otite média 21.

Para a dentição permanente, a literatura também descreve grande variação na prevalência para os três tipos de defeitos de esmalte, tal como ocorre na dentição decídua 4,22,23,24.

Li et al. 15 relatam que, mesmo usando um mesmo índice para verificação dos defeitos de esmalte, como, por exemplo, o DDE, este pode não fornecer comparações claras devido a sua própria complexidade e também por outros fatores experimentais, tais como o uso de diferentes fontes de luz, exame clínico dos dentes com secagem ou não, número de dentes ou superfícies examinadas, concentração de flúor na água de abastecimento público. Mesmo assim ainda é um índice descritivo das condições clínicas do esmalte mais comumente usado, pois não é requerido que a etiologia do tipo de defeito seja conhecida para se fechar um diagnóstico, podendo ser mais prático, apresentando maior possibilidade de comparação entre os estudos 19.

Os resultados deste estudo apontaram também uma maior prevalência de opacidades demarcadas do que hipoplasias, tanto na dentição decídua quanto na dentição permanente (Tabela 1), resultado esse que corrobora com outros estudos: num estudo realizado em Portugal 23, foi encontrada uma prevalência de 7,3\% e 7,1\% para opacidades demarcadas nas idades de 5 e 12 anos, respectivamente, enquanto que, para hipoplasia, as porcentagens foram de $0,3 \%$ e $0,9 \%$, respectivamente.

Ainda em relação à fluorose dentária, em um recente estudo epidemiológico de base nacional, Projeto SB Brasil ${ }^{9}$, foi encontrada uma prevalência de $9 \%$ dessa condição em crianças de 12 anos. Os maiores índices foram encontrados nas regiões Sudeste e Sul (em torno de 12\%), enquanto que os menores foram nas regiões Centro-Oeste e Nordeste (cerca de 4\%). Nos resultados ainda referentes a esse levantamento nacional, quando analisamos somente dados do Estado de São Paulo (Região Sudeste), em regiões com água fluoretada, a prevalência encontrada para fluorose 
aos 12 anos foi de $25 \%$, resultado esse semelhante ao encontrado em Indaiatuba $(26,2 \%)$.

Quando se comparou a prevalência dos três tipos de defeitos de esmalte (hipoplasia, opacidade demarcada e fluorose dentária) na dentição decídua entre os indivíduos livres de cárie (ceo = 0) e indivíduos com cárie (ceo $>0$ ), a diferença foi estatisticamente significante para os três tipos de defeitos (Figura 1).

As crianças que tinham opacidade demarcada na dentição decídua apresentaram chance quase duas vezes maior de ter cárie $(1,30<\mathrm{OR}$ $<2,95)$. Já as crianças com hipoplasia na dentição decídua apresentaram chance cinco vezes maior de ter cárie $(2,44<\mathrm{OR}<9,73)$. Nas crianças com fluorose na dentição decídua, foi encontrada chance quase duas vezes maior dessas terem cárie $(1,03<\mathrm{OR}<3,00)$, resultado esse não esperado, ressaltando a importância de que novos estudos sejam conduzidos, pois outros fatores, como, por exemplo, uma droga de amplo uso pediátrico (Amoxicilina), podem estar induzindo a formação de dentes com aspectos clínicos semelhantes aos observados na fluorose dentária 21 , superestimando a prevalência da fluorose dentária quando, na verdade, as alterações diagnosticadas não foram provocadas pela ingestão crônica de fluoreto.

Os resultados deste estudo corroboram os de Casanova-Rosado et al. 22 que também encontraram, nos seus resultados, relação entre experiência de cárie e presença de defeitos de esmalte (OR $=4,92$; IC95\%: 2,45-9,88), sendo que indivíduos com defeitos de esmalte apresentaram chance de ter cárie quase cinco vezes maior do que indivíduos sem defeitos de esmalte.

Outro estudo realizado na cidade de Head Start, Connecticut, Estados Unidos 1, teve como objetivos determinar a prevalência de cárie e defeitos de esmalte, bem como verificar se havia associação entre defeitos e cárie na dentição decídua. A população estudada era composta, em grande parte, (98\%) por americanos hispânicos e africanos. Como essa população apresentava homogeneidade para nível sócio-econômico, localização geográfica e prevalência de cárie, procurou-se encontrar um outro fator que pudesse estar relacionado à maior prevalência de cárie nessa população. Encontrou-se associação entre a presença de defeitos de esmalte e uma maior experiência de cárie, ou seja, quem tinha defeito de esmalte apresentava chance duas vezes maior de ter cárie (a prevalência de defeitos de esmalte encontrada foi de $49 \%$ ). Porém, quando essa mesma comparação foi feita para a dentição permanente neste presente estudo da cidade de Indaiatuba, as hipoplasias e as opacidades demarcadas mostraram uma associação com cárie dentária ( $\mathrm{p}=0,009$ e $\mathrm{p}=0,012$, respectivamente), e a fluorose dentária não se relacionou com a presença de cárie. $\mathrm{O}$ fato de as crianças terem seus dentes examinados sem escovação prévia e sob luz natural, no presente estudo, pode ter produzido resultados subestimados, assim esses resultados devem ser comparados com cautela.

Seow ${ }^{3}$, em um estudo sobre diagnóstico clínico de defeitos de esmalte, relata que alguns tipos de defeitos na dentição decídua podem ser detectados mais dificilmente do que na dentição permanente por causa de sua presença mais sutil. Essas formas mais suaves da presença de defeitos de esmalte poderiam ser resultado de várias questões. Primeiro, o ambiente intra-uterino poderia estar protegendo o esmalte formado durante o período pré-natal e, dessa forma, mudanças mínimas são observadas nas coroas dos dentes decíduos. Por exemplo, em doenças renais congênitas, o esmalte dental formado no período intra-uterino não é afetado geralmente devido à proteção normal fisiológica materna em relação ao infante. Segundo, devido ao período mais curto de formação do esmalte e uma espessura mais fina da camada de esmalte, as opacidades podem tornar-se menos evidentes. Além disso, a aparência mais esbranquiçada do esmalte decíduo pode dificultar o discernimento de lesões mais suaves, tais como as da fluorose dentária classificadas como leves. Terceiro, por razões ainda não claras cientificamente, existe uma expressão menor de certas doenças familiares e genéticas na dentição decídua. Assim, os resultados deste presente estudo sobre a presença de associação positiva entre fluorose e cárie na dentição decídua e ausência dessa associação para dentição permanente devem ser avaliados sobre a visão de possível subestimativa de prevalência de fluorose na dentição decídua, até porque o fato de a prevalência dessas alterações ter sido maior na dentição permanente pode estar representando a possibilidade de não associação entre cárie e fluorose nesta idade dos 12 anos.

Os defeitos de esmalte são um importante indicador de risco de cárie 25 porque a ultra-estrutura do esmalte, que provavelmente é anormal, também é mais susceptível à desmineralização 26 .

Os dados deste estudo apontam para a importância da discriminação dos tipos de defeitos de esmalte em levantamentos epidemiológicos, salientando-se que a presença destes defeitos, ou seja, as hipoplasias e as opacidades demarcadas, indicaram uma maior chance de as crianças virem a ter cárie dentária, tanto na dentição decídua como na permanente. Entretanto, como as taxas de prevalência de defeitos de esmalte, tanto na dentição decídua quanto na dentição per- 
manente, encontradas na literatura apresentam uma grande amplitude de 1,9\% a 99\% 27, dependendo da população estudada, dos dentes examinados e dos critérios utilizados para os diagnósticos, está claro que outros estudos devem ser realizados para que as causas das alterações sejam melhores investigadas, considerando que o esmalte, além de ser um importante marcador biológico da história passada da doença cárie, também pode fornecer subsídios e indicativos para investigar outras doenças e fatores de exposição ambientais.

\section{Resumo}

Os objetivos deste estudo foram verificar a prevalência de hipoplasia, opacidade demarcada e fluorose dentária em escolares nas dentições decídua e permanente e verificar a associação entre a presença desses defeitos de esmalte e a cárie dentária. A amostra foi de 624 pré-escolares de 5 anos e 309 escolares de 12 anos. A prevalência de cárie dentária foi avaliada por meio dos índices ceod e CPOD, e a prevalência dos defeitos de esmalte, com o índice DDE. A fluorose dentária foi medida pelos critérios do índice de Dean. O teste do qui-quadrado foi usado para verificar a associação e a razão de chances, analisando a força e a direção da associação. Aos cinco anos de idade, em crianças com experiência de cárie, houve associação positiva entre cárie dentária e defeitos de esmalte. Entretanto, na dentição permanente, apenas a hipoplasia e a opacidade demarcada foram associadas à cárie. Os resultados deste estudo indicaram maior chance de crianças virem a ter cárie, tanto na dentição decídua como na permanente, na presença de defeitos de esmalte, porém mais estudos são necessários para a comprovação dessa associação.

Hipoplasia do Esmalte Dentário; Fluorose Dentária; Cárie Dentária; Saúde Bucal

\section{Colaboradores}

R. H. S. Hoffmann, M. L. R. Sousa e S. Cypriano participaram da concepção do estudo, da análise dos resultados, assim como da revisão do manuscrito. M. L. R. Sousa e S. Cypriano coordenaram o processo de calibração (treinamento para a realização da pesquisa). R. H. S. Hoffmann realizou a redação geral do texto. 


\section{Referências}

1. Montero MJ, Douglass JM, Mathieu GM. Prevalence of dental caries and enamel defects in Connecticut Head Start children. Pediatr Dent 2003; 25:235-9.

2. Ribeiro AG, Oliveira AF, Rosemblat A. Cárie precoce na infância: prevalência e fatores de risco em pré-escolares, aos 48 meses, na cidade de João Pessoa, Paraíba, Brasil. Cad Saúde Pública 2005; 21:1695-700

3. Seow KW. Clinical diagnosis of enamel defects: pitfalls and practical guidelines. Int Dent J 1997; 47:173-82.

4. World Health Organization. Oral health surveys: basic methods. $4^{\text {th }}$ Ed. Geneva: World Health Organization; 1997.

5. Kanchanakamol U, Tuongratanaphan S, Lertpoonvilaikul W, Chittaisong C, Pattanaporn D, Navia JM, et al. Prevalence of developmental enamel defects and dental caries in rural pre-school Thai children. Community Dent Health 1996; 13:204-7.

6. Li Y, Navia JM, Bian JY. Caries experience in deciduous dentition of rural Chinese children 3-5 years old in relation to the presence or absence of enamel hypoplasia. Caries Res 1996; 30:8-15.

7. Milgrom P, Riedy CA, Weinstein P, Tanner AC, Manibusan LB. Dental caries and its relationship to bacterial infection hypoplasia, diet and oral hygiene in 6 to 36 month-old children. Community Dent Oral Epidemiol 2000; 28:295-306.

8. Ellwood RP, O'Mullane DM. Association between dental enamel opacities and dental caries in a North Wales population. Caries Res 1994; 28 : 383-7.

9. Ministério da Saúde. Projeto SB Brasil 2000: condições de saúde bucal da população brasileira no ano 2000. Manual do examinador. Brasília: Ministério da Saúde; 2001.

10. Suckling GW, Nelson DGA, Patel MJ. Macroscopic and scanning electron microscopic appearance and hardness values of developmental defects in human permanent tooth enamel. Adv Dent Res 1989; 3:219-33.

11. Slayton RL, Warren JJ, Kanellis MJ, Levy SM, Eslam M. Prevalence of enamel hypoplasia and isolated opacities in the primary dentition. Pediatr Dent 2001; 23:32-6.

12. Psoter WJ, Reid BC, Katz RV. Malnutrition and dental caries: a review of the literature. Caries Res 2005; 39:441-7.

13. Suckling GW, Elliott DC, Thurley DC. The production of developmental defects of enamel in the incisor teeth of penned sheep resulting from induced parasititism. Arch Oral Biol 1983; 28:393-9.

14. Jontell M, Linde A. Nutritional aspects on tooth formation. World Rev Nutr Diet 1986; 48:114-36.
15. Li Y, Navia JM, Bian JY. Prevalence and distribution of development enamel defects in primary dentition of Chinese children 3-5 years old. Community Dent Oral Epidemiol 1995; 23:72-9.

16. Cypriano S, Sousa MLR, Rihs LB, Wada RS. Saúde bucal dos pré-escolares, Piracicaba, Brasil, 1999. Rev Saúde Pública 2003; 37:247-53.

17. Fomon SJ, Ekstrand J, Ziegler EE. Fluoride intake and prevalence of dental fluorosis: trends in fluoride intake with special attention to infants. J Public Health Dent 2000; 60:131-9.

18. Whelton H, Crowley E, O'Mullane DM, Donaldson M, Kelleher V, Cronin M. Dental caries and enamel fluorosis among the fluoridated and non-fluoridated populations in the Republic of Ireland in 2002. Community Dent Health 2004; 21:37-44.

19. Mackay TD, Thomson WM. Enamel defects and dental caries among Southland children. N Z Dent J 2005; 101:35-43.

20. Whelton HP, Ketley CE, McSweeney F, O'Mullane DM. A review of fluorosis in the European Union: prevalence, risk factors and aesthetic issues. Community Dent Oral Epidemiol 2004; 32 Suppl 1: 9-18.

21. Hong L, Levy SM, Warren JJ, Dawson DV, Bergus GR, Wefel JS. Association of amoxicillin use during early childhood with developmental tooth enamel defects. Arch Pediatr Adolesc Med 2005; 159:943-8.

22. Casanova-Rosado AJ, Medina-Solis CE, CasanovaRosado JF, Vallejos-Sanchez AA, Maupomé G, Ávila-Burgos L. Dental caries and associated factors in México schoolchildren aged 6-13 years. Acta Odontol Scand 2005; 63:245-51.

23. Almeida CM, Petersen PE, Andre SJ, Toscano A. Changing oral health status or 6- and 12-year-old schoolchildren in Portugal. Community Dental Health 2003; 20:211-6.

24. Downer MC, Blinkhorn AS, Holt RD, Wight C, Attwood D. Dental caries experience and defects of dental enamel among 12-year-old children in north London, Edinburgh, Glasgow and Dublin. Community Dent Oral Epidemiol 1994; 22:283-5.

25. Seow KW. Biological mechanism of early childhood caries. Community Dent Oral Epidemiol 1998; 26:8-27.

26. Suckling GW, Herbison GP, Brown RH. Etiological factors influencing the prevalence of developmental defects of dental enamel in nine-year-old New Zealand children participating in a health and developmental study. J Dent Res 1987; 66:1466-9.

27. Lunardelli SE, Peres MA. Prevalence and distribution of developmental enamel defects in the primary dentition of pre-school children. Pesqui Odontol Bras 2005; 19:144-9.

Recebido em 16/Jan/2006

Versão final reapresentada em 22/Mai/2006

Aprovado em 02/Jun/2006 